(C) 2018 IEEE. Personal use of this material is permitted. Permission from IEEE must be obtained for all other uses, in any current or future media, including reprinting/republishing this material for advertising or promotional purposes, creating new collective works, for resale or redistribution to servers or lists, or reuse of any copyrighted component of this work in other works. 


\title{
Selective Recruitment of Arm Motoneurons in Nonhuman Primates Using Epidural Electrical Stimulation of the Cervical Spinal Cord
}

\author{
Beatrice Barra*, Camille Roux*, Mélanie Kaeser, Giuseppe Schiavone, Stéphanie P. Lacour, Jocelyne \\ Bloch, Grégoire Courtine, Eric M. Rouiller, Eric Schmidlin** and Marco Capogrosso**
}

\begin{abstract}
Recovery of reaching and grasping ability is a crucial function to recover from cervical spinal cord injury (SCI). Epidural electrical stimulation (EES) has shown promising results in improving limbs motor control after SCI in various animal models and in humans. In particular, spatiotemporal alternation of stimulation bursts during movement restored skilled locomotion in rats and Rhesus monkeys with SCI. By mimicking spinal segments activation patterns, these refined stimulation protocols could be pivotal also for the recovery of functional reaching and grasping movements. Indeed, skilled arm control requires complex coordinated activations of multiple arm/hand muscles that might be difficult to achieve with simple continuous stimulation protocols. Here we studied the activation patterns of motorpools in the cervical spinal cord during a reaching and grasping task in primates, for the design of spatiotemporal cervical epidural stimulation protocols. Based on such activation maps we propose a simple spatiotemporal stimulation protocol mimicking the natural synergistic muscle activation during upper limb movements.
\end{abstract}

\section{INTRODUCTION}

Recovery of reaching and grasping ability is critical for people suffering from cervical spinal cord injury (SCI). Today, no effective treatment exists for this condition. Systems based on functional electrical stimulation (FES) of the forearm muscles have restored simple hand and arm movements in non-human primates [1] and humans [2,3] with complete paralysis. Nonetheless, control of the threedimensional arm movements underlying daily life activities requires a complex coordination of multiple arm and hand muscles which represents a significant engineering challenge [4]. Moreover, FES recruits muscle fibers in an unnatural way, leading to fatigue and non-natural movement patterns.

*, ** These authors equally contributed to this work

B. Barra is with the Department of Neuroscience and Movement Science, Platform of Translational Neuroscience, University of Fribourg. Fribourg, Switzerland (corresponding author: Phone +41 21300 8769; email: beatrice.barra@unifr.ch ).

C. Roux, M. Kaeser, E. Schmidlin, E. Rouiller and M. Capogrosso, are with Department of Neuroscience and Movement Science, Platform of Translational Neuroscience, University of Fribourg. Fribourg, Switzerland (e-mail: $\quad$ camille.roux@unifr.ch, $\quad$ eric.schmidlin@unifr.ch, eric.rouiller@unifr.ch, marco.capogrosso@unifr.ch ).

G. Schiavone and S. Lacour are with the Center for Neuroprosthetics, School of Engineering, Institutes of Microengineering and Bioengineering, Ecole Polytechnique Federale de Lausanne (EPFL), Lausanne, Switzerland (e-mail: giuseppe.schiavone@epfl.ch, stephanie.lacour@epfl.ch).

G. Courtine is with the Center for Neuroprosthetics and Brain Mind Institute, School of Life Sciences, Ecole Polytechnique Federale de Lausanne (EPFL), Lausanne, Switzerland (e-mail: gregoire.courtine @epfl.ch).

J. Bloch is with the Centre Hospitalier Universitaire Vaudois (CHUV), Lausanne, Switzerland (e-mail: jocelyne.bloch@chuv.ch).
Therefore, while technically suited to restore fine grasping abilities, it may be unfit to produce smooth and sustained muscle forces during gravity compensated three-dimensional reaching tasks. Numerous works studied whether intraspinal microstimulation (ISMS) could remedy the limitations of FES. The delivery of micro currents to specific grey-matter sites could activate spinal cord circuitry, which allowed the recruitment of coordinated muscle contractions [5]. Indeed, ISMS trains delivered in the cervical spinal cord elicited functional arm and hand movements in a macaque [6]. These works illustrated the possibility of controlling coordinated movements of multiple muscles through a small number of stimulation channels. However, ISMS has shown limited specificity and highly variable outcomes, especially for the recruitment of extensors muscles in primates [7, 8]. Therefore, the designing of specific stimulation patterns that are needed to elicit a complete coordinated reaching and grasping movement seems daunting. In a different context, epidural electrical stimulation (EES) has shown promising results in improving weight-bearing locomotion and voluntary movements in animal models and in humans with SCI [9-12]. In particular, spatiotemporal alternation of EES bursts have been proven effective in modulating and controlling a broad spectrum of leg movements in rats and nonhuman primates [13-15]. It has been proposed that these protocols, mimicking motoneuron recruitment patterns during movement, exploit dynamic properties of spinal circuits through the modulation of proprioceptive feedback circuits [16], thereby enabling the control of synergistic muscle groups. Moreover, recent results have shown the ability of continuous EES protocols to improve voluntary grasping functions in patients with spinal cord injury [12]. These results open the intriguing possibility to exploit similar stimulation protocols for the recovery of three-dimensional reaching and grasping movements. Here we studied the spatiotemporal patterns of motoneuron activation in the cervical spinal cord during a reach and grasp drawer task [17] in a Macaca Fascicularis. We then characterized the specificity of EES applied at the cervical spinal cord using a customized implant in the same animal during an anesthetized terminal procedure. Finally, we show that natural spatiotemporal patterns of motoneurons activation can be reproduced using a combination of few stimulation sites.

\section{METHODS}

In order to define a spatiotemporal EES strategy, we conducted two experiments. First, we inspected whether a reaching and grasping task would imply a well-defined spatial and temporal pattern of motoneuron activation (Experiment A). Second, we assessed whether it was possible to selectively recruit groups of functionally activated muscles 
when delivering EES from a multi active-site epidural electrode (Experiment B) with the aim of reproducing the motoneurons activation patterns underlying reaching and grasping movements.

\section{A. Animal and surgical procedures}

The experimental protocol was approved by the cantonal (Fribourg) and federal (Swiss) veterinary authorities (authorization No. 2014_42E_FR). Experiments were performed on a female macaque monkey (Mk-CA, Macaca Fascicularis, 12 years, $5.9 \mathrm{Kg}$ ). In Experiment A, Mk-CA was trained to perform a reach and grasp drawer task [18-20] while Intramuscular electromyographic (EMG) activity from $\mathrm{n}=8$ muscles was recorded. Experiment $\mathrm{B}$ was conducted during a terminal procedure before euthanasia while the animal was kept in deep anaesthesia (induction with ketamine + dormicum + fentanyl, and maintaining with intravenous infusion of propofol). Oxygen supply was provided by means of a nasal tube and heart rate, body temperature and blood oxygenation were continuously monitored throughout.

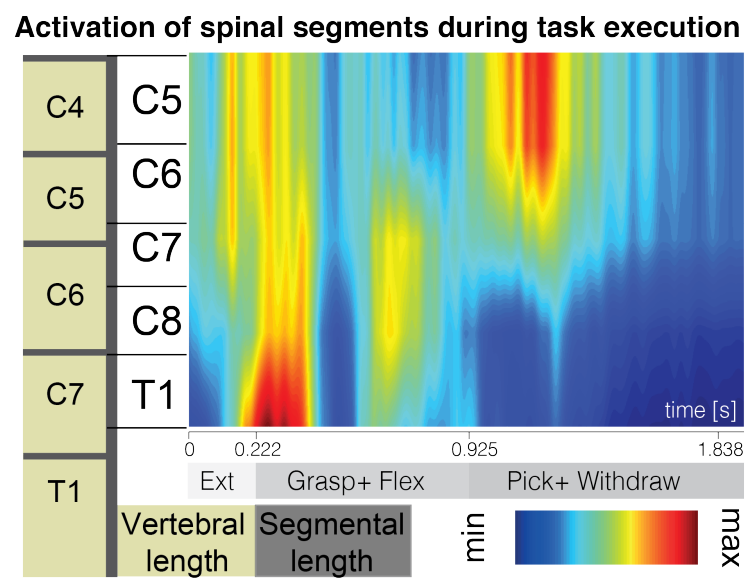

Figure 1. Spatiotemporal motoneurons recruitment map dur0ing the execution of a reach and grasp drawer task. The task comprises three phases: an extension (Ext), a grasping + flexion (Grasp+Flex) and the reward picking ( Pick + withdraw). The color code shows the level of activation of the moroneurons located in the spinal segments from C5 to T1.

\section{B. Identification of spinal cord activation patterns during movement}

We studied the activation patterns of the cervical spinal cord segments during a reach and grasp drawer task in primates. Anatomical studies [21] documented the rostrocaudal distribution of motoneurons innervating different arm and hand muscles. The behavioral task consisted in the reaching and opening of a drawer, containing a food reward. The MkCA was trained to rest the hand on a pad while waiting for a starting cue. Once ready, the subject had to single-handedly reach for the drawer knob, open the drawer and pick the contained reward (food pellet) contained therein. EMG activity was recorded from eight chronically implanted muscles during the repeated execution of the task. We automatically extracted intra-movement phases: the extension phase (from movement onset to drawer handling); grasping/pulling of the drawer until complete opening; and reward picking. We then projected the recorded EMG activity to the anatomical location of the corresponding motoneurons using information in extracted from [21] in order to reconstruct the spatiotemporal activation patterns of arm and hand motoneurons during each of these phases (Fig. $1)$.

\section{Electrode specific recruitment curves}

A customized spinal implant based on previously reported technology [23] and comprising $n=7$ independent electrodes was surgically inserted into the epidural space of the cervical spinal cord from an entry point obtained through a laminectomy at the vertebral level T1. Single pulse stimulation at different current amplitudes was delivered from each electrode of the spinal implant while intramuscular EMG activity was recorded from $n=8$ muscles.

\section{Electrode position Electrode specific on spinal cord}
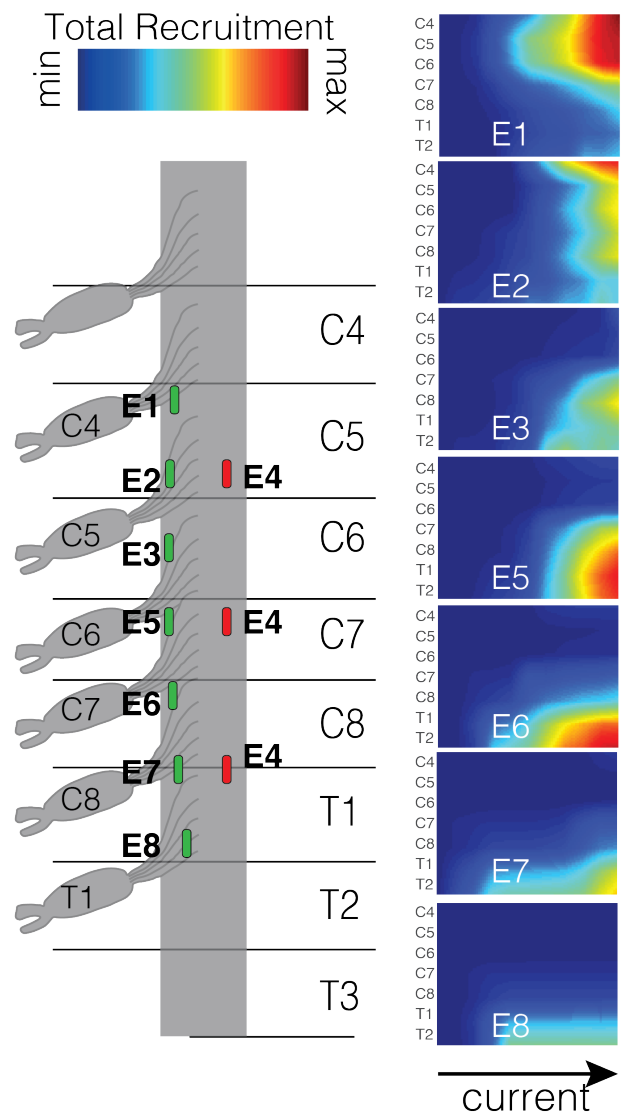

Figure 2. Spatial motoneuron recruitment patterns at increasing

stimulation currents, for each stimulation electrode. Active sites are numbered from E1 to E8 and their relative positioning on the spinal cord is shown on the left. E4 comprises 3 shorted electrodes.

In addition, we extracted the spatial pattern of motoneuron recruitment in response to each single pulse of stimulation (Fig. 2). The mapping of the muscles on the spinal cord segments relied on the distribution of motoneurons described in [21]. We then calculated the level of muscle recruitment over the stimulation current amplitudes for each active site separately and we computed recruitment curves. Muscle 
specificity of i-th muscle was quantified using the selectivity index $\mathrm{S}_{\mathrm{i}}$ [22] defined as:

$$
S_{i}=\mu_{i}-\frac{1}{(m-1)} \sum_{j=1, j \neq i}^{m} \mu_{i}
$$

where $\mu_{\mathrm{i}}$ is the activation level of the $\mathrm{i}^{\text {th }}$ muscle and $\mathrm{m}$ is the total number of muscles.

\section{Design of stimulation protocol}

In order to design an EES strategy that would reproduce the spatiotemporal motoneuron recruitment pattern underlying reaching and grasping movements, we generated time sequences of spatial motoneuron activation patterns elicited by single pulses of EES delivered from the different active sites of the epidural electrode array. Specifically, the active sites and current amplitudes used at the time step were selected as the most suited EES parameters to reproduce the activation level of each segment at this specific moment, as identified from the spatiotemporal map underlying the reach and grasp drawer task.

\section{RESUlts}

\section{A. Identification of spinal cord activation patterns during movement (Experiment A)}

The spatiotemporal maps of arm and hand motoneuron activation in the cervical spinal cord during reaching and grasping movements revealed a well-defined pattern of activation over time (Fig. 1, average $\mathrm{n}=35$ repetitions). During the extension phase, the segments that are mainly activated are those innervating the deltoid and triceps muscles (C4 and C5). The transition to the second phase was associated with the activation of extensor hand muscles (innervated in T1) together with flexor muscles of the forearm (innervated at C7). An activation of the T1 spinal segment anticipated the withdraw phase, as the animal was picking the food reward. An activation of the most rostral segments ensued, which was linked to the activation of the deltoid muscle. This activation pattern presented a series of well separated subsequent spinal cord activation hotspots during the task. We concluded that the reach and grasp drawer tasks could be represented by a well-defined temporal pattern of motoneuron recruitment.

\section{B. Muscle responses induced by cervical EES}

The recruitment curves computed for the different active sites highlighted a good spatial selectivity of the epidural implant. Fig. 2 shows spinal maps reconstructed for all the electrodes, associated to their position on the spinal cord. Each stimulation site activates preferentially the spinal segments that are located in close proximity. Fig. 3 shows muscle recruitment curves and selectivity indices of the active sites E1, E2 and E6. These three active sites enabled the selective activation of arm/hand flexors, arm/hand extensors and digit muscles, respectively. We conclude that EES of the cervical spinal cord may be able to access specific groups of functionally activated muscles.

\section{Design of stimulation protocols}

Fig. 4 shows a reconstructed spatiotemporal map elaborated from the single pulse spatial motoneuron responses. We combined responses from the three electrodes (E1, E2 and E6) through a temporal sequence and current amplitude modulation (1 to $2 \mathrm{~mA}$ ) that matched spatiotemporal map measured during movement. The resulting map showed a succession of spatially defined hotspots that were qualitatively similar to those found in the task-specific map.

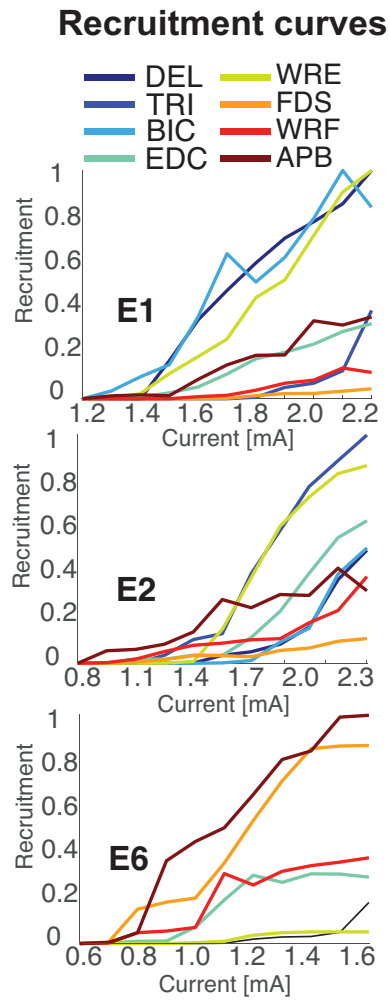

\section{Active site specific selectivity index}

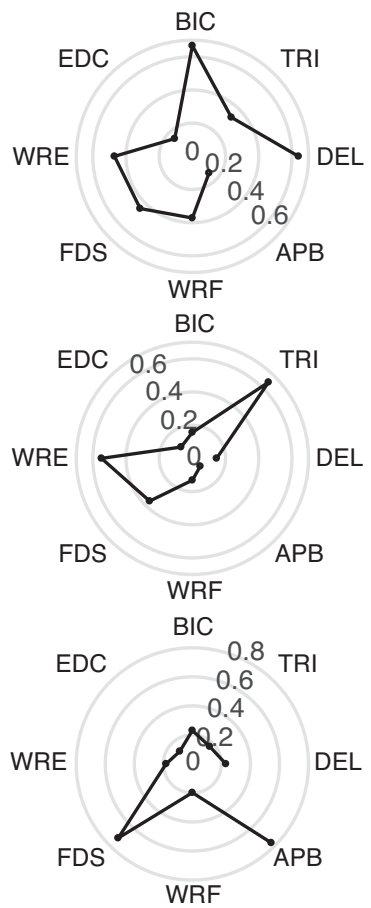

Figure 3. Recruitment curves and selectivity indeces are shown for all the muscles and for the three epidural electrode active sites E1, E2 and E6 (from top to bottom). Muscles shown: deltoid (DEL), biceps (BIC), triceps (TRI), extensor digitorium communis (EDC), wrist extensor ( WRE), flexor digitorium superficialis (FDS), wrist flexor (WRF), abductor pollicis brevis (APB).

\section{CONCLUSIONS}

In this study we inspected whether a reach and grasp drawer task implies a well-defined spatial and temporal pattern of motoneuron activation. Successively, we assessed whether it was possible to selectively recruit groups of synergistic muscles when delivering EES from a multi active-site epidural electrode with the aim of reproducing the motoneurons activation patterns underlying reaching and grasping movements. The reproduced activation map qualitatively matched spatiotemporal map measured during the execution of the task (Fig. 4). Therefore, we concluded that it is possible to define selective EES protocols of the cervical spinal cord reproducing coordinated activation of arm and hand muscles during functional reaching and grasping movements in nonhuman primates. These combined 
results show encouraging data for the design of selective EES stimulation protocols for improving upper limb function in people with tetraplegia.

\section{Spinal activation map during task}

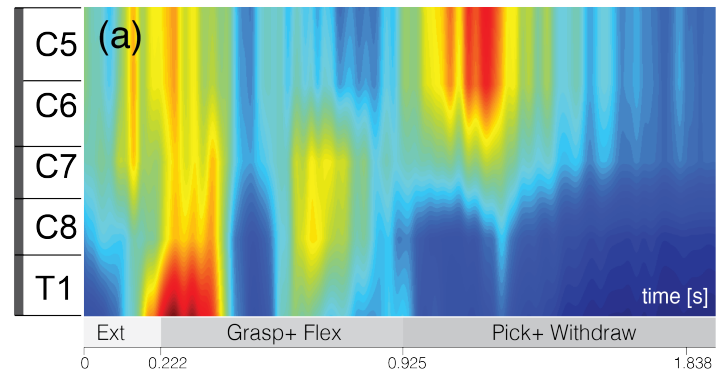

Reconstructed spinal activation map

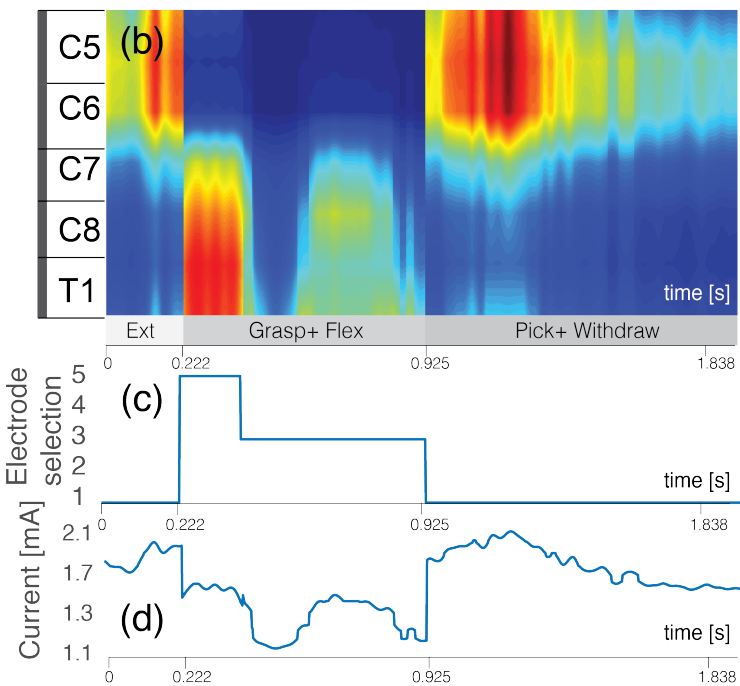

Figure 4. The task-specific spatiotemporal activation was reproduced selecting the spinal segments activation obtained from single pulse EES delivered from 3 electrodes. Current amplitudes were chosen to mimic at best the activation pattern obtained during the task. From top to bottom: (a) the original activation map represents the activation of the spinal segments from $\mathrm{C} 5$ to $\mathrm{T} 1$; (b) the reconstructed activation map shows the activation of the spinal segments that would be induced by the stimulation delivered from 3 electrodes; (c) the curve shows which active sites are selected over time during the task to reproduce the spinal activation map; (d) the curve indicates the amplitude of the stimulation current for the chosen electrode over time.

\section{ACKNOWLEDGMENTS}

This work is supported by: a grant from the Wings for Life to M.C. the Ambizione program of the Swiss National Science Foundation (SNSF) to M.C., the NCCR Robotics of the SNSF, the University of Fribourg and the Wyss Center for Bio and Neuro Engineering; the European Union's Horizon 2020 research and innovation programme under the Marie Skłodowska- Curie grant agreement No. 665667, and an Industrial Grant-Agreement between GTX Medical and the University of Fribourg.

\section{REFERENCES}

[1] Ethier, C., et al., Restoration of grasp following paralysis through brain-controlled stimulation of muscles. Nature, 2012. 485(7398): $\mathrm{p}$. 368-71.
[2] Friedenberg, D.A., et al., Neuroprosthetic-enabled control of graded arm muscle contraction in a paralyzed human. Sci Rep, 2017. 7(1): p. 8386.

[3] Ajiboye, A.B., et al., Restoration of reaching and grasping movements through brain-controlled muscle stimulation in a person with tetraplegia: a proof-of-concept demonstration. The Lancet, 2017. 389(10081): p. 1821-1831.

[4] Popovic, M.R., D.B. Popovic, and T. Keller, Neuroprostheses for grasping. Neurological Research, 2002. 24(5): p. 443-452.

[5] Schouenborg, J., Action-based sensory encoding in spinal sensorimotor circuits. Brain Res Rev, 2008. 57(1): p. 111-7.

[6] Zimmermann, J.B., K. Seki, and A. Jackson, Reanimating the arm and hand with intraspinal microstimulation. J Neural Eng, 2011. 8(5): p. 054001.

[7] Moritz, C.T., et al., Forelimb movements and muscle responses evoked by microstimulation of cervical spinal cord in sedated monkeys. J Neurophysiol, 2007. 97(1): p. 110-20.

[8] Gaunt, R.A., et al., Intraspinal microstimulation excites multisegmental sensory afferents at lower stimulus levels than local alpha-motoneuron responses. J Neurophysiol, 2006. 96(6): p. 29953005.

[9] Angeli, C.A., et al., Altering spinal cord excitability enables voluntary movements after chronic complete paralysis in humans. Brain, 2014. 137(Pt 5): p. 1394-409.

[10] Carhart, M.R., et al., Epidural spinal-cord stimulation facilitates recovery of functional walking following incomplete spinal-cord injury. IEEE Trans Neural Syst Rehabil Eng, 2004. 12(1): p. 32-42.

[11] Hofstoetter, U.S., et al., Periodic modulation of repetitively elicited monosynaptic reflexes of the human lumbosacral spinal cord. J Neurophysiol, 2015. 114(1): p. 400-10.

[12] Lu, D.C., et al., Engaging Cervical Spinal Cord Networks to Reenable Volitional Control of Hand Function in Tetraplegic Patients. Neurorehabil Neural Repair, 2016. 30(10): p. 951-962.

[13] Wenger, N., et al., Closed-loop neuromodulation of spinal sensorimotor circuits controls refined locomotion after complete spinal cord injury. ScienceTranslationalMedicine, 2014. 6(255): p. 255ra133.

[14] Wenger, N., et al., Spatiotemporal neuromodulation therapies engaging muscle synergies improve motor control after spinal cord injury. Nat Med, 2016. 22(2): p. 138-45.

[15] Capogrosso, M., et al., A brain-spine interface alleviating gait deficits after spinal cord injury in primates. Nature, 2016. 539(7628): p. 284288.

[16] Moraud, E.M., et al., Mechanisms Underlying the Neuromodulation of Spinal Circuits for Correcting Gait and Balance Deficits after Spinal Cord Injury. Neuron, 2016. 89(4): p. 814-28.

[17] Pirondini, E., et al., Evaluation of the effects of the Arm Light Exoskeleton on movement execution and muscle activities: a pilot study on healthy subjects. J Neuroeng Rehabil, 2016. 13: p. 9.

[18] Chatagny, P., et al., Distinction between hand dominance and hand preference in primates: a behavioral investigation of manual dexterity in nonhuman primates (macaques) and human subjects. Brain Behav, 2013. 3(5): p. 575-95

[19] Schmidlin, E., et al., Behavioral assessment of manual dexterity in non-human primates. J Vis Exp, 2011(57): 3258.

[20] Kaeser, M., et al., Variability of manual dexterity performance in nonhuman primates (Macaca Fascicularis). International Journal of Comparative Psychology, 2014. 27(2): p. 295-325.

[21] Jenny, A.B. and J. Inukai, Principles of Motor Organization of the Monkey Cervical Spinal Cord. The Journal of Neuroscience, 1983. 3(3): p. 567-575.

[22] Raspopovic, S., M. Capogrosso, and S. Micera, A computational model for the stimulation of rat sciatic nerve using a transverse intrafascicular multichannel electrode. IEEE Trans Neural Syst Rehabil Eng, 2011. 19(4): p. 333-44.

[23] Minev, I.R., et al., Electronic dura mater for long-term multimodal neural interfaces. Science, 2015. 347(6218): p. 159-163. 